Check for updates

Cite this: Chem. Commun., 2017, 53,8502

Received 5th May 2017,

Accepted 26th June 2017

DOI: $10.1039 / \mathrm{c} 7 \mathrm{cc} 03478 d$

rsc.li/chemcomm

\section{A mechano-responsive supramolecular metal- organic framework (supraMOF) gel material rich in ZIF-8 nanoplates $\dagger$}

\author{
Abhijeet K. Chaudhari (D) and Jin-Chong Tan (D)*
}

\begin{abstract}
We report an unconventional ZIF-8 based supramolecular MOF (supraMOF) material, which is straightforwardly synthesised by leveraging the high concentration reaction (HCR) approach. Akin to traditional low-molecular-weight gels (LMWG), we show that mechano-stimulus responsive behaviour can be achieved through a reversible and fast sol-gel conversion mechanism. Remarkably, the supraMOF gel consists of 2D nanoplates of ZIF-8 with a reduced crystal symmetry.
\end{abstract}

The chemistry beyond that of molecules, known as 'supramolecular chemistry ${ }^{1}$ has witnessed a rapid growth over the past two decades. ${ }^{2}$ Notably, the traditional candidates in the field of supramolecular science, featuring the self-assembly of small molecules comprising purely organic compounds, have been superseded by exotic and very diverse families of hybrid (organic-inorganic) molecular assemblies and composite systems. ${ }^{3}$ Self-assembly methodologies that yield functional gel materials have attracted significant interest owing to their potential applications in medicine,${ }^{4}$ energy, ${ }^{5}$ and environmental sustainability. ${ }^{6}$ Within this broad class of soft matters, metalcontaining gel materials termed 'metallogels" ${ }^{7}$ could offer additional controls for tuning the structural and physico-chemical properties for many targeted applications. ${ }^{8}$ Metal-containing metallo-gelators studied to date can basically be divided into two main categories: (a) solvent immobilisation attributed to metalcoordination effects ${ }^{9}$ and (b) solvent immobilisation conferred by metal complex species used for constructing metallogels. ${ }^{7 c, 3 b}$ Recently, researchers have also focused on metal-organic gels (MOGs), ${ }^{10}$ which generally contain continuous chains of alternating metal and organic molecules to enable immobilisation of solvents. ${ }^{11}$

Multifunctional Materials \& Composites (MMC) Laboratory, Department of Engineering Science, University of Oxford, Parks Road, Oxford, OX1 3PJ, UK. E-mail: jin-chong.tan@eng.ox.ac.uk

$\dagger$ Electronic supplementary information (ESI) available: Video showing the mechano-stimulus response. Methods, rheology data, SEM and TEM images, AFM characterisation, thermogravimetric analysis. See DOI: 10.1039/c7cc03478d
An innovative implementation of the basic principle of continuous chain formation of metal-organic building blocks has opened the door to the recent development of smart supramolecular gels, ${ }^{12}$ containing porous 3D scaffolding of metal-organic frameworks (MOFs). ${ }^{13}$ We termed these materials 'supraMOF', as they are derived from supramolecular gel selfassemblies coexisting with in situ generated MOF entities. Our recent study on the HKUST-1 based supraMOF has revealed promising sol-gel reversibility, affecting its chemical structure useful for tuning a range of physical properties including the electrical conductivity. ${ }^{12 a}$ The literature on MOF compounds has proliferated to cover a very wide and diverse spectrum of applications. ${ }^{14}$ Despite their vast potential utilities, MOF science in combination with self-assembled supramolecular materials is, hitherto, a largely unexplored research topic. One exciting possibility is to yield 'smart' supramolecular materials ${ }^{12 a, c}$ by leveraging the tunability of MOF compounds, to attain stimuliresponsive behaviours towards magnetism, light, sound waves, mechanical stresses, temperature, electricity, etc. Moreover, sol-gel systems will offer new possibilities for facile processing and affordable deposition of functional films towards engineering of sensors, actuators and microelectronic devices. ${ }^{15}$

In this communication, we report the discovery of a new candidate of supraMOF gel material built from the same chemical constituents as for a topical zeolitic imidazolate framework (ZIF) with sodalite topology: $\mathrm{ZIF}-8\left[\mathrm{Zn}(\mathrm{mIm})_{2} ; \mathrm{mIm}=2\right.$-methylimidazolate]. ${ }^{16}$ Notably, this unconventional ZIF-8 based supraMOF exhibits exceptional mechano-stimulation response, via a rapid sol-gel transformation bestowed upon it by its highly reversible mechanical properties in cyclic stress loading.

The ZIF-8 supraMOF was synthesised by adopting the 'high concentration reaction' (HCR) approach. ${ }^{12 a}$ This involves high concentrations of mIm and $\mathrm{Zn}\left(\mathrm{NO}_{3}\right)_{2}$ (see Table 1 , reaction A), but prior to mixing the mIm organic linker solution in $N, N$-dimethylformamide (DMF) solvent to the $\mathrm{Zn}$ (II) metal ion solution (also in DMF), the former was first deprotonated by taking an equivalent molar concentration of trimethylamine base $\left(\mathrm{NEt}_{3}\right)$. Upon combining the above solution reactants, we 
Table 1 Series of reactions $(A-E)$ to investigate the effects of concentration on the framework structure of ZIF-8. Note: DMF volume was divided $50: 50$ between $\mathrm{Zn}\left(\mathrm{NO}_{3}\right)_{2}$ and $\mathrm{mlm}$

\begin{tabular}{lclll}
\hline Reaction & $\begin{array}{l}\text { DMF (total) } \\
(\mathrm{mL})\end{array}$ & $\begin{array}{l}\mathrm{Zn}\left(\mathrm{NO}_{3}\right)_{2} \\
(\mathrm{mmol})\end{array}$ & $\begin{array}{l}\mathrm{mIm} \\
(\mathrm{mmol})\end{array}$ & $\begin{array}{l}\mathrm{NEt}_{3} \\
(\mathrm{mmol})\end{array}$ \\
\hline A (Gel) & 6 & 3 & 12 & 12 \\
B & 25 & 3 & 12 & 12 \\
C & 100 & 3 & 12 & 12 \\
D & 300 & 3 & 12 & 12 \\
E & 500 & 3 & 12 & 12 \\
\hline
\end{tabular}

observed that a white pre-gel first "material" containing fibre-like morphologies formed immediately, which then quickly converted into a gel phase within just a few seconds. Sonication for a further 5 seconds resulted in the formation of a mechanically stable gel phase. After sonication, the sample container was inverted to test for gelation (by inversion test), and we found that, similar to the conventional low-molecular-weight gels (LMWG), this gel material can resist gravity by refusing to flow downwards.

Interestingly, this candidate of ZIF-8 supraMOF exhibited rapid sol-gel phase transition upon application of an external mechanical stimulus (stress). Vigorous shaking of the sealed glass vial containing the gel material quickly transformed it into a sol phase, which could easily revert back to a stable gel phase simply by allowing the sol unperturbed for about 5 seconds. The real-time sol $\rightleftharpoons$ gel recovery can be seen in the video clip provided as a part of the ESI, $\dagger$ which demonstrates the fast phase reversibility in a matter of seconds.

To gain detailed insights into gel stability and the sol-gel conversion phenomenon, dynamic rheological measurements were performed using controlled parameters as shown in Fig. 1. It can be seen in Fig. 1(a) that the oscillatory frequency sweep between $0.1 \mathrm{~Hz}$ and $100 \mathrm{~Hz}$ showed a relatively steady behaviour for the storage modulus $\left(G^{\prime}\right)$ and loss modulus $\left(G^{\prime \prime}\right)$, without any strong dependence on the frequency until beyond $\sim 30 \mathrm{~Hz}$. After which, the magnitude of $G^{\prime}$ suddenly fell while $G^{\prime \prime}$ began to increase; this behaviour signifies the collapse of an integrated network of the gel phase. Likewise, the distinct increase of the loss tangent $\left(\tan (\delta)=G^{\prime \prime} / G^{\prime}\right)$ versus frequency data above $\sim 30 \mathrm{~Hz}$ is evident of an occurrence of the gel-to-sol phase transformation. We have determined that $G^{\prime}$ is an order of magnitude higher than $G^{\prime \prime}$, indicating that the ZIF-8 supraMOF can be considered as a stable gel material unperturbed. Fig. 1b shows the dynamic strain sweep measurements performed from 0.1 to $100 \%$ strain at $0.1 \mathrm{~Hz}$, to characterise the stability of the gel against phase change under an incremental shear strain. a)
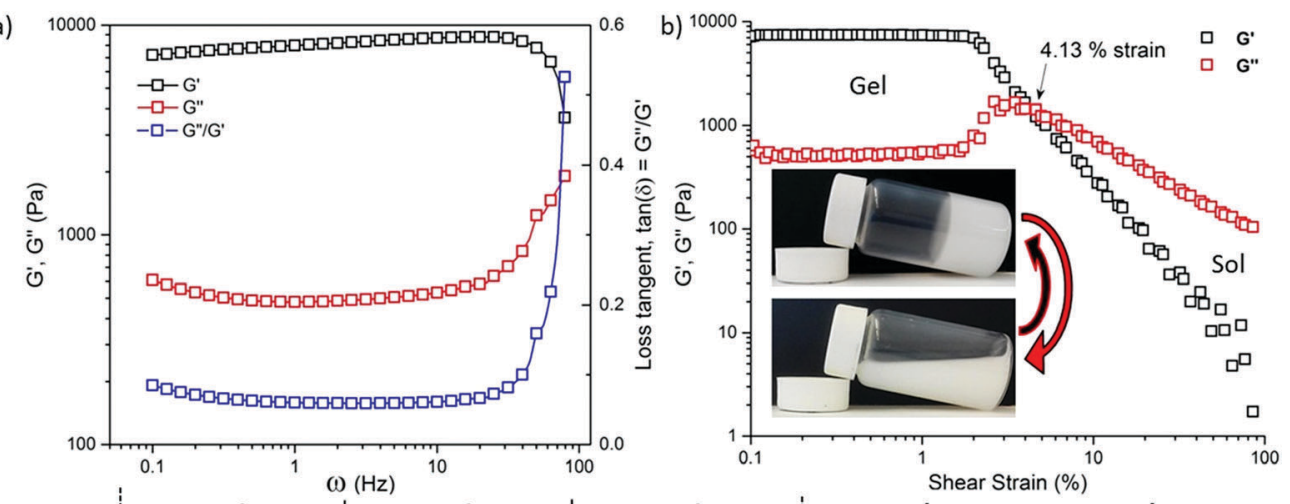

c)

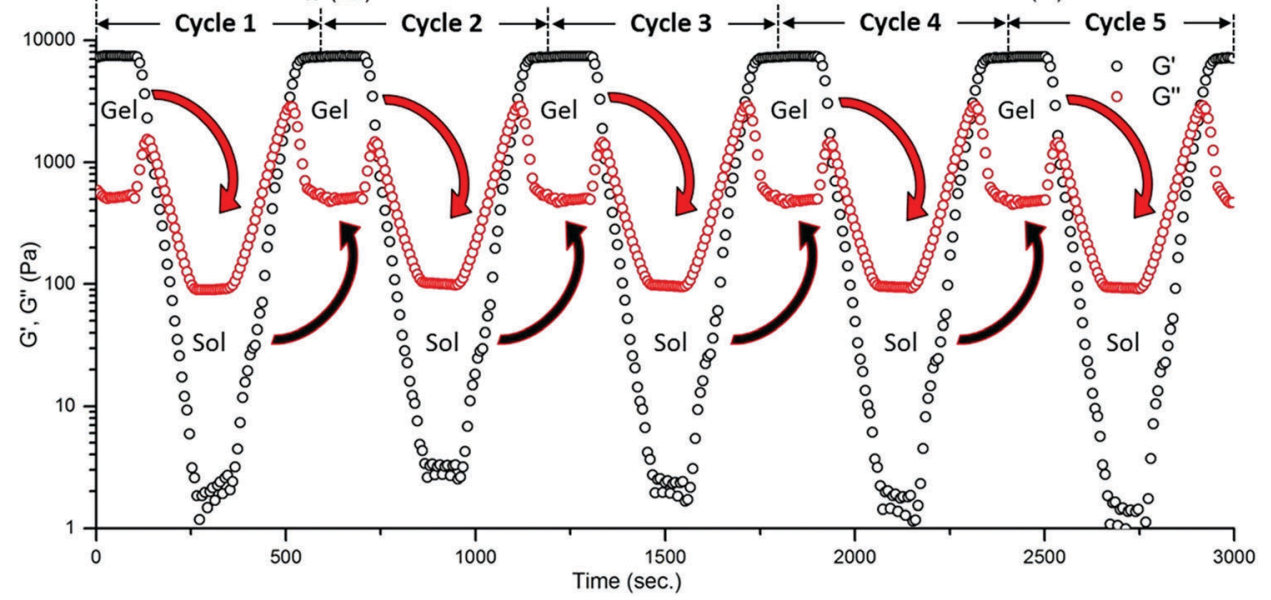

Fig. 1 (a) Oscillatory frequency-sweep rheological experiments for the ZIF-8 based supraMOF gel tested under a constant applied shear strain of $0.1 \%$. $G^{\prime}$ and $G^{\prime \prime}$ are the storage and loss moduli, respectively. Loss tangent, $\tan (\delta)=G^{\prime \prime} / G^{\prime}$, data indicating a phase change at above $\sim 30 \mathrm{~Hz}$. (b) Dynamic strain sweep measurements established the yield point at $\sim 4 \%$, at which the gel converts into sol. Inset: photos showing reversible sol-gel conversion. (c) Mechano-stimulus response of the ZIF- 8 supraMOF material tested by performing a cyclic dynamic strain-sweep experiment (consecutive 5 cycles are shown), demonstrating its capability to transform reversibly between gel $\rightleftharpoons$ sol with highly reproducible mechanical properties. 
Horizontal profiles of $G^{\prime}$ and $G^{\prime \prime}$ between 0.1 and $\sim 2 \%$ shear strain were found to start becoming strain dependent with an increase in the strain. The $G^{\prime}$ and $G^{\prime \prime}$ profiles coincided at $\sim 4 \%$, marking the yield point of the gel. Subsequently, the magnitude of $G^{\prime \prime}$ became much greater than that of $G^{\prime}$ between shear strains of $\sim 5$ and $100 \%$, clearly this meant that the energy dissipation has exceeded the energy storage because the stable gel phase has collapsed into a sol suspension (see Fig. 1b inset).

By imposing a cyclic (load-unload) mechanical stimulus, we have characterised the phase reversibility of the ZIF-8 supraMOF material as shown in Fig. 1c, so as to establish its thixotropic nature under a cyclic shear strain set between $0.1 \% \rightleftharpoons 100 \%$. We found that the results in Fig. $1 \mathrm{~b}$ are in fact reversible, that is, upon decreasing the shear strain from $100 \%$ to $0.1 \%$ the sol recovered to the gel form evidenced by $G^{\prime}>G^{\prime \prime}$ (storage $>$ loss). Such gel $\rightleftharpoons$ sol reversibility tests were performed for multiple consecutive cycles, for example, shown in Fig. $1 \mathrm{c}$ are data representing the profiles obtained by allowing $150 \mathrm{~s}$ for the recovery of gel from a collapsed sol phase. Furthermore, the cyclic stress experiments revealed precisely how the complex viscosity can be very reversibly tuned, over a wide range of $10^{2}-10^{4} \mathrm{~Pa} \mathrm{~s}$ as effected by a recoverable sol-gel transformation step (see Fig. S2 and S3 in the ESI $\dagger$ ).

To study the origin of the mechano-responsive behaviour (Fig. 1), we carried out a systematic materials characterisation study to correlate the observed property with its underlying material structure. A gel sample prepared on a piece of glass substrate by layering a thin layer of the material was imaged under a scanning electron microscope (SEM) to characterise its microstructure. The SEM micrographs obtained are shown in Fig. 2a (and Fig. S4 in the ESI $\dagger$ ), where the gel material forms what appeared to be a densely packed cellular-like phase, very unlike the typical fibrous network ${ }^{10 d, 17}$ found in conventional gel materials. To further understand the supraMOF gel constitution, we used copious amounts of DMF and methanol to break down (disintegrate) the gel phase, liberating an abundance of 'fine particles' that were then isolated by centrifugation $(8000 \mathrm{rpm})$. Surprisingly, transmission electron microscopy (TEM) revealed that the isolated particles resembled the two-dimensional (2D) morphologies of nanoplates (Fig. $2 \mathrm{~b}$ and Fig. S5-S7, ESI $†$ ). We confirmed the nanoplate geometry via atomic force microscopy (AFM) as presented in Fig. 2(c and d), where the averaged nanoplate thickness was found to be of the order of $10 \mathrm{~s} \mathrm{~nm}$ (Fig. S8-S12 and Table S2) and with an aspect ratio (i.e. width/ thickness) of at least $\sim 5$. Intriguingly the mechano-responsive gel phase comprises nanoplates of distorted ZIF-8, whose crystal structure is elucidated below.

The bulk aggregates of nanoplates harvested from the supraMOF gel phase transformed into a monolith after drying at $110{ }^{\circ} \mathrm{C}$. This monolith was carefully ground into a fine powder and then characterised using powder X-ray diffraction (PXRD). Fig. 3a shows that its PXRD pattern has sharp peaks matching the ideal simulated XRD pattern of ZIF-8, but with the appearance of a few broadened extra peaks suggesting the reduction of the crystal symmetry. To investigate this effect, we have carried out a systematic study by varying the reactant

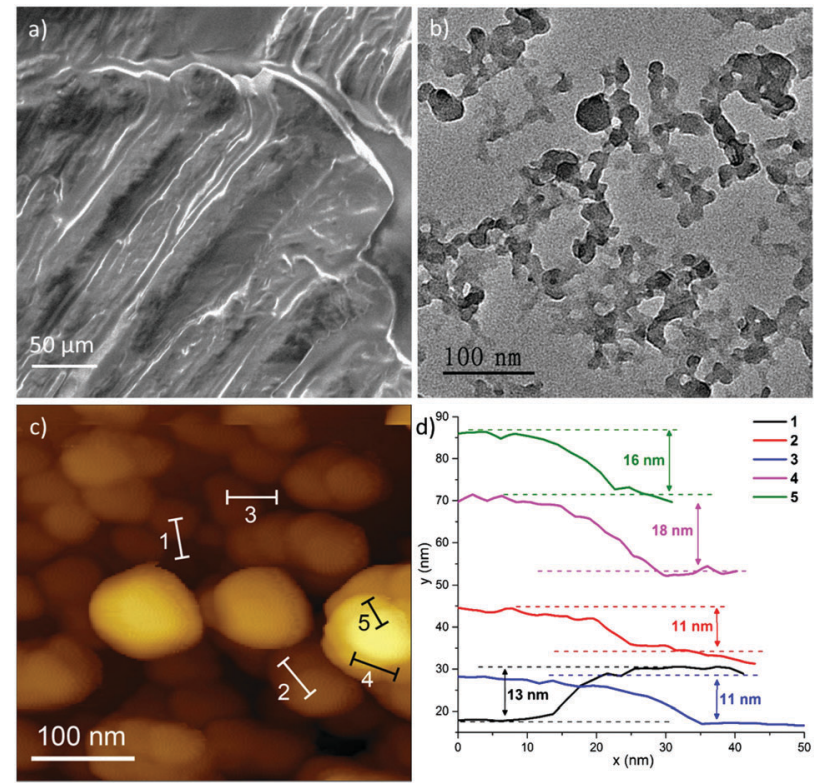

Fig. 2 (a) SEM image of the dried supraMOF gel material. (b) TEM image of the nanoplates obtained after breaking down the gel material into fine powder particles. (c) AFM topography image revealing the 2D morphology of (larger) isolated nanoplates, and (d) their individual thickness profiles of ca. 10-20 nm corresponding to the specific regions marked in (c).

concentrations in accordance with Table 1 . Specifically, different reactions using $\mathrm{mIm}$ and $\mathrm{Zn}$ (II) were performed by maintaining the same synthetic parameters apart from the total volume of the DMF solvent used, which was varied from $6 \mathrm{~mL}$ (for highly concentrated reactions) to $500 \mathrm{~mL}$ (very diluted reaction conditions). The products obtained from the five reactions were washed and dried prior to PXRD (Fig. 3a), showing a systematic reduction in intensity of the extra peaks with a greater solvent content. This result is supported by the systematic colour change of the samples detectable by the naked eye (Fig. 3a inset); we reasoned that additional guest species (e.g. $\mathrm{NEt}_{3}, \mathrm{DMF}$ ) become trapped in the framework from concentrated reactions which decompose upon heating of the compound producing a yellowish powder ( $v s$. a whiter powder of ZIF-8).

To determine the salient changes of the structural parameters of ZIF-8, the PXRD data from the reaction of A160 (gel) were utilised for Pawley structural refinement (see the ESI, $\dagger$ Table S3). The refinement results in Fig. 3(b) show that the high-symmetry (cubic) structure of ZIF-8 has reduced to a lowsymmetry (triclinic) crystal structure $\left(R_{\mathrm{wp}}=6.42 \%\right)$ for the nanoplates extracted from the ZIF-8 supraMOF gel. Cell dimensions of the cubic ZIF-8 structure were found to have been modified from $a, b, c=17.086 \AA$ to $a=17.46 \AA, b=17.12 \AA$, $c=16.95 \AA$, and the unit cell angles changed from $\alpha, \beta, \gamma=90^{\circ}$ to $\alpha=89.01^{\circ}, \beta=89.50^{\circ}, \gamma=88.19^{\circ}$. Moreover, thermogravimetric analysis (TGA) of these compounds detected changes in the thermal stability of the framework material when being synthesised under concentrated conditions (Fig. S13 and S14, ESI $\uparrow$ ). Unlike the thermal stability of the conventional ZIF-8 powder till $\sim 350{ }^{\circ} \mathrm{C}$, the nanoplates comprising reduced symmetry ZIF-8 (derived from supraMOF) have a relatively lower thermal 

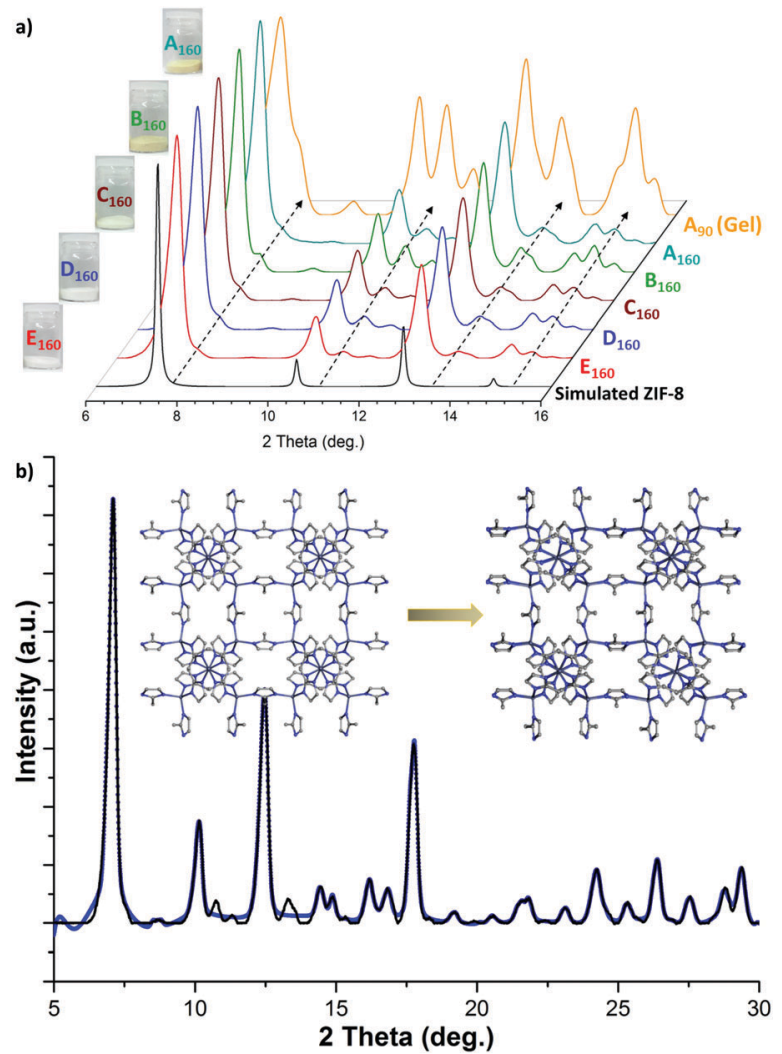

Fig. 3 (a) PXRD patterns of samples derived from the reactions labelled $A-E$ in Table 1, where the subscripts denote the drying temperature of either $90{ }^{\circ} \mathrm{C}$ or $160{ }^{\circ} \mathrm{C}$. Sample colours obtained (shown to the left of XRD spectra) revealed the apparent variations between the $A$ to $E$ reaction products. Dotted arrows designate the positions of the extra diffraction peaks due to occluded guests. (b) Pawley refinement method to determine the lattice geometry of the distorted ZIF- 8 framework from the experimental PXRD data of the A160 sample. Inset: structural changes from the (standard) cubic cell of ZIF-8 to a triclinic distorted ZIF-8 structure.

stability of up to $\sim 250{ }^{\circ} \mathrm{C}$, which can be ascribed to the decomposition of the entrapped guest species.

To conclude, we have demonstrated the efficacy of the highconcentration reaction (HCR) strategy to derive a previously unreported ZIF-8-based supraMOF gel material. Surprisingly, this supraMOF gel contains an abundance of ZIF-8 nanoplates with only $\sim 10 \mathrm{~s} \mathrm{~nm}$ thickness, completely dissimilar to the conventional ZIF-8's rhombic dodecahedral crystals ubiquitous in the literature. Exceptionally, this ZIF-8 supraMOF gel is mechano-stimuli responsive, exhibiting fast sol-gel conversions and perfectly reversible mechanical properties subject to a multi-cyclic strain loading. Our current study not only presents the first discovery of a ZIF-type gel material, but also describes the simple synthetic methodology that may be widened to explore its applicability for other well-studied MOFs to yield supramolecular stimuli-responsive systems and to achieve rare $2 \mathrm{D}$ morphologies.

We thank the Samsung GRO and EPSRC RCUK (EP/N014960/1 and EP/K031503/1) for research funding, and the Research Complex at Harwell (RCaH) for the provision of TEM and TGA facilities. We are grateful to Dr Gavin Stenning and Dr Marek Jura for access to PXRD at the R53 Materials Characterisation Laboratory in ISIS RAL, Oxfordshire.

\section{Notes and references}

1 (a) J. M. Lehn, Angew. Chem., Int. Ed., 1990, 29, 1304-1319; (b) F. M. Menger, Proc. Natl. Acad. Sci. U. S. A., 2002, 99, 4818-4822. 2 (a) L. J. Prins, J. Huskens, F. de Jong, P. Timmerman and D. N. Reinhoudt, Nature, 1999, 398, 498-502; (b) O. A. Scherman, Nat. Chem., 2009, 1, 524-525; (c) T. Aida, E. W. Meijer and S. I. Stupp, Science, 2012, 335, 813-817; (d) C. Hansell, Nat. Chem., 2014, 6, 459.

3 (a) L. E. Buerkle and S. J. Rowan, Chem. Soc. Rev., 2012, 41, 6089-6102; (b) A. Y. Tam and V. W. Yam, Chem. Soc. Rev., 2013, 42, 1540-1567; (c) S. Y. Dong, J. Y. Yuan and F. H. Huang, Chem. Sci., 2014, 5, 247-252; (d) Q. Lin, B. Sun, Q. P. Yang, Y. P. Fu, X. Zhu, Y. M. Zhang and T. B. Wei, Chem. Commun., 2014, 50, 10669-10671.

4 (a) M. E. Byrne, K. Park and N. A. Peppas, Adv. Drug Delivery Rev., 2002, 54, 149-161; (b) J. A. Foster, M. O. Piepenbrock, G. O. Lloyd, N. Clarke, J. A. Howard and J. W. Steed, Nat. Chem., 2010, 2, 1037-1043.

5 (a) J. H. Wu, Z. Lan, J. M. Lin, M. L. Huang, S. C. Hao, T. Sato and S. Yin, Adv. Mater., 2007, 19, 4006-4011; (b) Y. Kim, M. Kathaperumal, V. W. Chen, Y. Park, C. Fuentes-Hernandez, M. J. Pan, B. Kippelen and J. W. Perry, Adv. Energy Mater., 2015, 5, 1500767.

6 D. Mohan and C. U. Pittman, Jr., J. Hazard. Mater., 2007, 142, 1-53.

7 (a) K. Murata, M. Aoki, T. Nishi, A. Ikeda and S. Shinkai, Chem. Commun., 1991, 1715-1718; (b) B. G. Xing, M. F. Choi and B. Xu, Chem. - Eur. J., 2002, 8, 5028-5032; (c) A. Y. Tam, K. M. Wong, G. Wang and V. W. Yam, Chem. Commun., 2007, 2028-2030.

8 (a) M. O. M. Piepenbrock, N. Clarke and J. W. Steed, Langmuir, 2009, 25, 8451-8456; (b) M. O. M. Piepenbrock, G. O. Lloyd, N. Clarke and J. W. Steed, Chem. Rev., 2010, 110, 1960-2004; (c) T. Tu, W. Fang, X. Bao, X. Li and K. H. Dotz, Angew. Chem., Int. Ed., 2011, 50, 6601-6605; (d) W. Miao, L. Zhang, X. Wang, H. Cao, Q. Jin and M. Liu, Chem. - Eur. J., 2013, 19, 3029-3036.

9 W. J. Gee and S. R. Batten, Chem. Commun., 2012, 48, 4830-4832.

10 (a) Q. Wei and S. L. James, Chem. Commun., 2005, 1555-1556; (b) S. K. Nune, P. K. Thallapally and B. P. McGrail, J. Mater. Chem., 2010, 20, 7623-7625; (c) F. Yang, Z. Lin, X. He, L. Chen and Y. Zhang, J. Chromatogr. A, 2011, 1218, 9194-9201; (d) S. Samai and K. Biradha, Chem. Mater., 2012, 24, 1165-1173; (e) S. Saha, G. Das, J. Thote and R. Banerjee, J. Am. Chem. Soc., 2014, 136, 14845-14851.

11 (a) J. Y. Zhang and C. Y. Su, Coord. Chem. Rev., 2013, 257, 1373-1408; (b) Y.-M. Zhang, X.-M. You, H. Yao, Y. Guo, P. Zhang, B.-B. Shi, J. Liu, Q. Lin and T.-B. Wei, Supramol. Chem., 2013, 26, 39-47.

12 (a) A. K. Chaudhari, I. Han and J. C. Tan, Adv. Mater., 2015, 27, 4438-4446; (b) B. Bueken, N. Van Velthoven, T. Willhammar, T. Stassin, I. Stassen, D. A. Keen, G. V. Baron, J. F. M. Denayer, R. Ameloot, S. Bals, D. De Vos and T. D. Bennett, Chem. Sci., 2017, 8, 3939-3948; (c) A. K. Chaudhari, H. J. Kim, I. Han and J. C. Tan, Adv. Mater., 2017, DOI: 10.1002/adma.201701463.

13 (a) B. F. Hoskins and R. Robson, J. Am. Chem. Soc., 1989, 111, 5962-5964; (b) O. M. Yaghi, G. M. Li and H. L. Li, Nature, 1995, 378, 703-706; (c) S. Kitagawa, R. Kitaura and S. Noro, Angew. Chem., Int. Ed., 2004, 43, 2334-2375; (d) J. C. Tan and B. Civalleri, CrystEngComm, 2015, 17, 197-198. 14 (a) G. Ferey, Chem. Soc. Rev., 2008, 37, 191-214; (b) V. Stavila, A. A. Talin and M. D. Allendorf, Chem. Soc. Rev., 2014, 43, 5994-6010; (c) M. G. Campbell, D. Sheberla, S. F. Liu, T. M. Swager and M. Dinca, Angew. Chem., Int. Ed., 2015, 54, 4349-4352; (d) A. K. Chaudhari, M. R. Ryder and J. C. Tan, Nanoscale, 2016, 8, 6851-6859.

15 (a) L. E. Kreno, K. Leong, O. K. Farha, M. Allendorf, R. P. Van Duyne and J. T. Hupp, Chem. Rev., 2012, 112, 1105-1125; (b) M. R. Ryder and J. C. Tan, Mater. Sci. Technol., 2014, 30, 1598-1612; (c) I. Stassen, N. Burtch, A. Talin, P. Falcaro, M. Allendorf and R. Ameloot, Chem. Soc. Rev., 2017, 46, 3185-3241.

16 (a) K. S. Park, Z. Ni, A. P. Cote, J. Y. Choi, R. Huang, F. J. Uribe-Romo, H. K. Chae, M. O'Keeffe and O. M. Yaghi, Proc. Natl. Acad. Sci. U. S. A., 2006, 103, 10186-10191; (b) J. C. Tan, B. Civalleri, C. C. Lin, L. Valenzano, R. Galvelis, P. F. Chen, T. D. Bennett, C. Mellot-Draznieks, C. M. ZicovichWilson and A. K. Cheetham, Phys. Rev. Lett., 2012, 108, 095502.

17 (a) K. Jayaramulu, F. Geyer, M. Petr, R. Zboril, D. Vollmer and R. A. Fischer, Adv. Mater., 2017, 29, 1605307; (b) R. C. T. Howe, A. P. Smalley, A. P. M. Guttenplan, M. W. R. Doggett, M. D. Eddleston, J. C. Tan and G. O. Lloyd, Chem. Commun., 2013, 49, 4268-4270. 